\title{
Schottky photodiode using submicron thick diamond epilayer for flame sensing
}

\author{
Y. Koide ${ }^{1, *}$, M. Y. Liao ${ }^{1, * *}$, J. Alvarez², M. Imura ${ }^{3}$, K. Sueishi ${ }^{4}$ and F. Yoshifusa ${ }^{4}$
}

The sensing of a flame can be performed by using wide-bandgap semiconductors, which offer a high signal-to-noise ratio since they only response the ultraviolet emission in the flame. Diamond is a robust semiconductor with a wide-bandgap of $5.5 \mathrm{eV}$, exhibiting an intrinsic solar-blindness for deep-ultraviolet (DUV) detection. In this work, by using a submicron thick boron-doped diamond epilayer grown on a type-Ib diamond substrate, a Schottky photodiode device structure- based flame sensor is demonstrated. The photodiode exhibits extremely low dark current in both forward and reverse modes due to the holes depletion in the epilayer. The photodiode has a photoconductivity gain larger than 100 and a threshold wavelength of $330 \mathrm{~nm}$ in the forward bias mode. $\mathrm{CO}$ and $\mathrm{OH}$ emission bands with wavelengths shorter than $330 \mathrm{~nm}$ in a flame light are detected at a forward voltage of $-10 \mathrm{~V}$. An alcohol lamp flame in the distance of $250 \mathrm{~mm}$ is directly detected without a focusing lens of flame light.

Keywords: Flame sensing; Boron-doped diamond; Schottky photodiode

Citation: Y. Koide, M. Y. Liao, J. Alvarez, M. Imura, K. Sueishi and F. Yoshifusa, "Schottky photodiode using submicron thick diamond epilayer for flame sensing", Nano-Micro Lett. 1, 30-33 (2009). doi: 10.5101/nml.v1i1.p30-33

Solar ultraviolet (UV) radiation with wavelength shorter than $280 \mathrm{~nm}$ (i.e. UV-C) is completely absorbed by the stratospheric ozone layer above the Earth's surface. An optical sensor, which selectively detects the deep-ultraviolet (DUV) light and remains blind to the light with wavelength larger than $280 \mathrm{~nm}$ is called a solar-blind DUV photosensor. Since the hydrocarbon flame has $\mathrm{CO}$ and $\mathrm{OH}$ emission bands with wavelength smaller than 350 $\mathrm{nm}$, such a DUV photosensor can be applied as a flame sensor even in the presence of solar radiation. The commercialized flame sensor is the photocathode tube with a high sensitivity to the light with wavelength ranging from 230 to $280 \mathrm{~nm}$. The disadvantages of the photocathode tube are the high operation voltage $(>400 \mathrm{~V})$, difficulty in sensitivity control by changing the bias voltage, readily deterioration under continuous DUV-light illumination, and a weak glass body. Thus, the development of solid-state flame sensors is mandatory in order to overcome these disadvantages.

There exist a few reports to demonstrate the flame sensors using semiconducting $\mathrm{SiC}$ and $\mathrm{Al}_{\mathrm{x}} \mathrm{Ga}_{1-\mathrm{x}} \mathrm{N}$ [1,2]. However, the drawbacks of these $\mathrm{SiC}$ and $\mathrm{AlGaN}$-based flame sensors are low responsivity to DUV light and low discrimination ratio of the responsivity between the DUV and visible lights. In addition, in some cases such as a spark in the welding process, no deterioration is essential to continuously detect the strong flame. Hence, an extremely robust material, which is stable under long-term or strong DUV illumination, is necessary for

${ }^{1}$ Sensor Materials Center (SenMC), National Institute for Materials Science (NIMS), 1-1 Namiki, Tsukuba, 305-0044 Ibaraki, Japan

2Laboratoire de Génie Electrique de Paris (UMR 8507 CNRS), Ecole Supérieure d'Electricité, Universités Paris VI et Paris XI, 11 rue Joliot-Curie Plateau de Moulon, F-91192 Gif-sur-Yvette Cedex, France

3International Center for Materials Nanoarchitectonics (MANA), National Institute for Materials Science (NIMS), 1-1 Namiki, Tsukuba, 305-0044 Ibaraki, Japan ${ }^{4}$ ANTEC Co. 116-3 Mameda, Oku-cho, Setouch, 701-4254 Okayama, Japan

*,**Corresponding author. Email: a) Koide.Yasuo@nims.go.jp, b) Meiyong.Liao@nims.go.jp 
flame sensing. Semiconducting diamond with a bandgap of 5.5 $\mathrm{eV}$ is an attractive material due to its weak sensitivity in the visible wavelength range and high sensitivity in the DUV range (i.e., it has solar blindness).

Intrinsic diamond shows a cut-off wavelength of $225 \mathrm{~nm}$ beyond the flame UV emission. It is basically difficult to use only thick intrinsic diamond for flame sensing. However, the submicron thin film technology and impurity engineering provide the opportunity to apply diamond to flame sensing. By using submicron thick boron-doped homoepitaxial diamond layers on type-Ib diamond substrates and proper device structures, it is able to tune the overall properties of diamond photodetectors [3]. For example, the dark current can be extremely low due to the depletion of free holes in the submicron epilayer [4]. The threshold wavelength of the diamond detector can be tailored to be larger than $225 \mathrm{~nm}$ [4-7]. The photoconductivity gain can be as larger as 100 upon DUV light illumination. Thus, the diamond photosensor developed is able to detect the $\mathrm{CO}$ and $\mathrm{OH}$ emission bands in the hydrocarbon flame.

In this work, we demonstrate the solid-state flame sensor by using the high- sensitivity diamond DUV photosensor. In addition, we show the flame sensing performance of the diamond photosensor.

The p-diamond epilayers were homoepitaxially grown on Ib-type nitrogen-containing diamond (100) substrates with a dimension of $2.5 \times 2.5 \times 0.5 \mathrm{~mm}$ by a microwave plasmaenhanced chemical vapor deposition technique. The growth conditions were described in the previous paper [5,6]. The thickness of the epilayer was about $0.5 \mu \mathrm{m}$, and the [B] in the epilayer and the $[\mathrm{N}]$ in the substrate were measured by secondary-ion mass spectroscopy to be mid $10^{15}$ and mid $10^{19}$ $\mathrm{cm}^{-3}$, respectively. Prior to fabricating the device, the surfaces of the epilayers were oxidized in a boiling acid solution of $\mathrm{H}_{2} \mathrm{SO}_{4}$ and $\mathrm{HNO}_{3}$ to remove the surface conductive hydrogenated layers. Planar-type Schottky photodiodes (SPD) with semi-transparent WC Schottky and annealed Ti/WC Ohmic contacts were fabricated on the epilayer [5,6]. A WC Schottky contact with a nominal thickness of 3-5 nm was deposited in a defined circle pattern with a diameter of $0.98 \mathrm{~mm}$. The interspacing between the Schottky and Ohmic contacts was $10 \mu \mathrm{m}$. Finally, the photodiode chip was packaged in a TO5-type can with a sapphire window.

The current-voltage characteristics were measured in a vacuum chamber at a pressure of $0.1 \mathrm{~Pa}$ by using a two-point probe method. The photoresponse properties were measured by illuminating monochromatic light with a wavelength between 210 and $630 \mathrm{~nm}$ using a 500-W xenon lamp. The incident light power was calibrated using a UV-enhanced Si photodiode. The time response of the SPD photodiode was measured by a digital oscilloscope. The flame sensing properties were measured using an alcohol lamp. Photoluminescence of the alcohol lamp flame was measured using the spectrometer system.

Figure 1 shows luminescence spectra of alcohol lamp flame and solar radiation through a room window glass. The major peaks of the flame are $\mathrm{OH}-$ and $\mathrm{H}$-related emissions at 310 and $590 \mathrm{~nm}$, and a shoulder band from 240 to $300 \mathrm{~nm}$ is observed in the DUV wavelength region [8]. In order to detect the flame optically under the existence of solar radiation, we have to develop the DUV photosensor with a large responsivity for

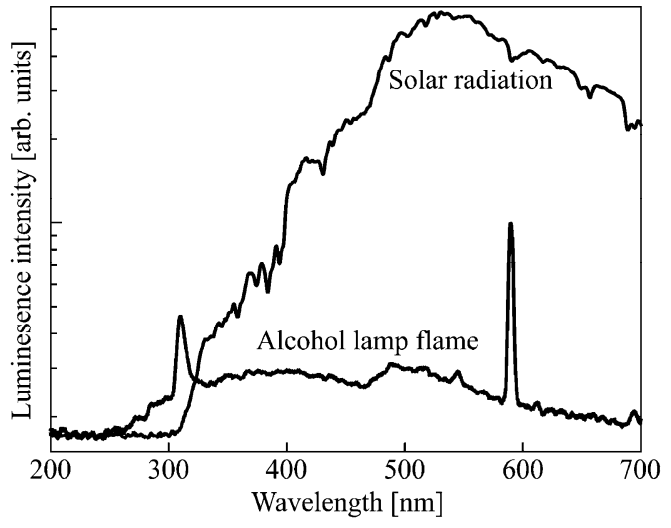

FIG. 1. Luminescent spectra of an alcohol lamp flame and solar radiation through a room window.

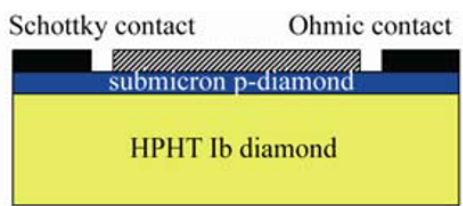

(a)

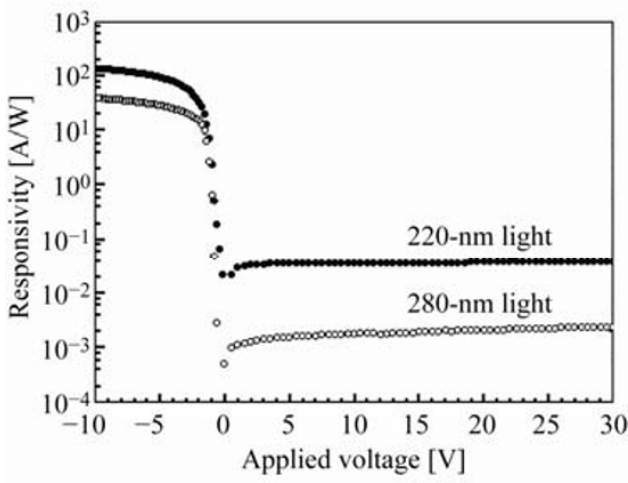

(b)

FIG. 2. (a) Schottky photodiode based on submicron boron-doped epilayer grown on a type-Ib HPHT diamond substrate and (b) responsivity of 220 and $280 \mathrm{~nm}$ lights as a function of applied voltage, where negative and positive applied voltages correspond to forward and reverse biasing modes, respectively. 
wavelengths lower than $300 \mathrm{~nm}$ and a large discrimination ratio between the DUV and visible light.

Figure 2(a) illustrates the Schottky photodiode by using submicron boron-doped p-type diamond grown on a HPHT type-Ib diamond substrate. Figure 2(b) depicts the responsivities of the diamond photosensor for 220 and 280 $\mathrm{nm}$-lights illumination as a function of applied bias voltage, where forward and reverse biases correspond to negative and positive voltages, respectively. The dark current of the photosensor was lower than $0.1 \mathrm{pA}$ in the forward and reverse bias modes. Since the nitrogen in the diamond was known to behave as a donor with thermal activation energy of $1.7 \mathrm{eV}$ [9], the high density of nitrogen in the Ib-type substrate is believed to deplete the hole in the thin epilayer, which results in the extremely low current even in the forward bias mode. Note that this leads to enhancement of the detectivity by using the Ib-type substrate. The voltage dependence of responsivity shows the clear rectifying properties, which indicates that the current transport is controlled by photo-generated holes. This is the characteristic feature of the diamond SPD as reported previously [5]. The responsivities as large as $136 \mathrm{~A} / \mathrm{W}$ for 220 $\mathrm{nm}$ light and $38 \mathrm{~A} / \mathrm{W}$ for $280 \mathrm{~nm}$ light is obtained in a forward bias voltage of $-10 \mathrm{~V}$. The corresponding quantum efficiency $\eta$, values are $7.7 \times 10^{4} \%$ for $220 \mathrm{~nm}$ light and $1.7 \times 10^{4} \%$ for 280 $\mathrm{nm}$ light, which indicates the photoconductivity gain property as reported previously [4,5]. The gain mechanism is explained by existence of electron trap with high capture rate and low emission rate, which provides the significant increment of the hole lifetime and the hole concentration [3,6]. This model is supported by the predominant current transport due to hole. Since we also observed the gain property of the MSM photoconductor at an applied voltage lower than $1 \mathrm{~V}$ [7], the interaction between the epilayer and the Ib-substrate is believed to be responsible for the kinetic gain mechanism.

Figure 3 shows time dependences of (a) responsivity of 220 $\mathrm{nm}$ light at $-10 \mathrm{~V}$, where curve (b) shows the $220 \mathrm{~nm}$ light pulse for comparison. The transient behavior to the $220 \mathrm{~nm}$ light of the current circled SPD is similar to the interdigitated SPD operated at forward biases [4]. The response time is evaluated to be as small as $5 \mathrm{~ms}$. The response time for $280 \mathrm{~nm}$ light pulse is absolutely the same as that for the $220 \mathrm{~nm}$ light. Note that the present SPD has the high DUV-sensitivity and the response speed enough to detect the flame.

The d.c. spectral response of the SPD at a forward bias of $-10 \mathrm{~V}$ is shown in Fig. 4. Differed from the reported diamond photodetectors [5,7], the threshold wavelength shifts from 270

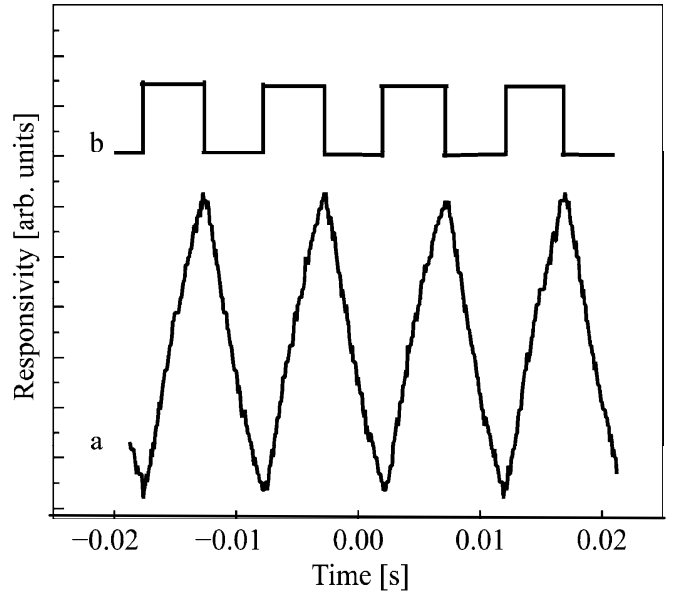

FIG. 3. (a) Transient behavior of the SPD to the pulsed $220 \mathrm{~nm}$ light at a forward bias of $-10 \mathrm{~V}$ and (b) the reference signal of the pulsed $220 \mathrm{~nm}$ light.

$\mathrm{nm}$ to around $330 \mathrm{~nm}$. This value does not change as the Schottky contact diameter increases from the previous $400 \mu \mathrm{m}$ to the current $980 \mu \mathrm{m}$ [4]. However, the visible-blind ratio $(210 / 400 \mathrm{~nm})$ increases greatly as the Schottky diameter increases. This value reaches up to around eight orders of magnitude in the present SPD due to the increased UV sensitivity. The cut-off wavelength and the high visible-blind ratio offer the current diamond SPD a potential candidate for flame sensing.

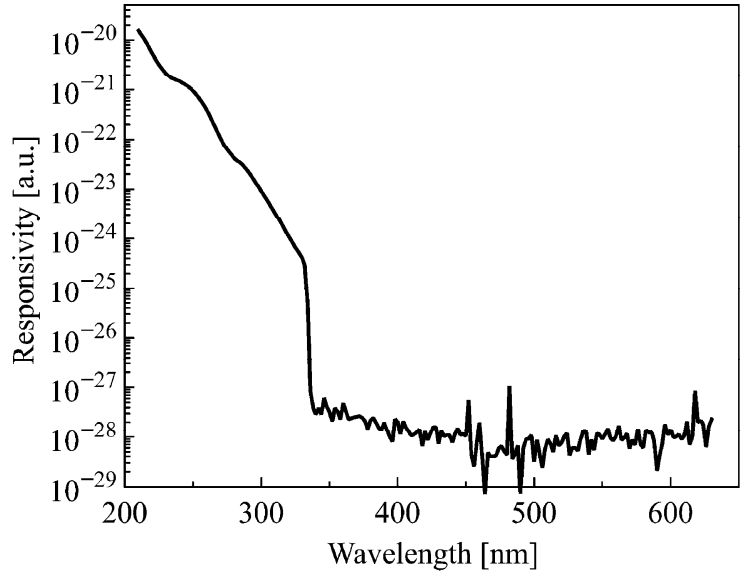

FIG. 4. Spectral response of the SPD at a forward bias of $-10 \mathrm{~V}$.

Figure 5 is a photograph of sensing the alcohol lamp flame by the diamond SPD under bulb light illumination background, where an inside photograph shows the photosensors packaged in the TO5 can. The diamond photosensor is set on the left-hand side at $250 \mathrm{~mm}$ in length apart from the alcohol lamp flame. The photosensor is able to detect the flame in the distance of $250 \mathrm{~mm}$ at a forward voltage of $-10 \mathrm{~V}$, and the photocurrent level is around $100 \mathrm{pA}$. Note that the flame can be detected without using a focusing lens. Since the threshold wavelength 


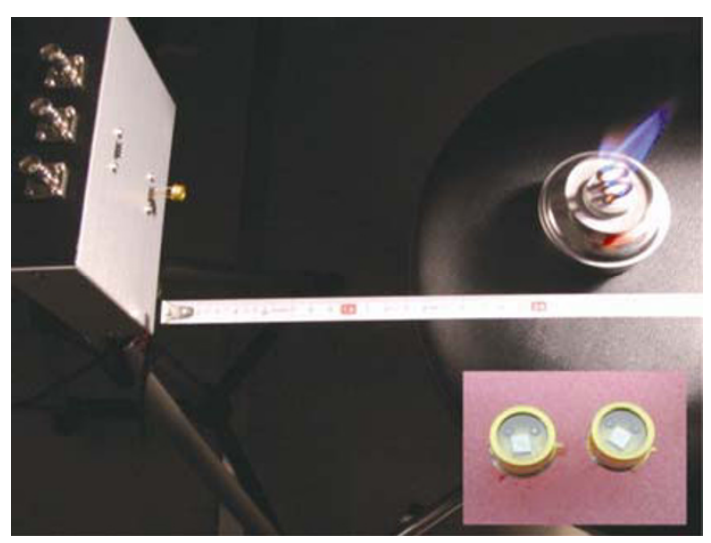

FIG. 5. Photograph demonstrating the diamond photosensor to detect the alcohol lamp flame.

of the present SPD was around $330 \mathrm{~nm}$, the $\mathrm{CO}$ and $\mathrm{OH}$ emission bands with wavelength shorter than $330 \mathrm{~nm}$ is believed to be predominantly detected. This is the distinct demonstration which is really capable of sensing the hydrocarbon flame using the diamond DUV photosensor.

In summary, by using a submicron thick boron-doped diamond epilayer on a type-Ib diamond substrate, the cut-off wavelength of the diamond SPD was extended to be around 330 $\mathrm{nm}$ at forward biases. By virtue of this property, we developed the flame sensor by using the diamond SPD packaged in the TO5 can with the sapphire window and demonstrated sensing the alcohol lamp flame. Although we did not use the focusing lens of DUV light, the photosensor was able to detect the flame reproducibly in the distance of $250 \mathrm{~mm}$. The development of the flame sensor opened a new application avenue for diamond photosensors.
This work was supported by Grant-in-Aid for Scientific Research in the Ministry of Education, Culture, Sports, Science and Technology of the Japanese Government (No. 18360341).

Received 11 November 2009; accepted 17 November 2009; published online 25 November 2009.

\section{References}

1. D. M. Brown, E. Downey, J. Kretchmer, G. Michon, E. Shu and D. Schneider, Solid-State Electron. 42, 755 (1998). doi:10.1016/S0038-1101(97)00260-8

2. A. Hirano, C. Pernot, M. Iwaya, T. Detchprohm, H. Amano and I. Akasaki, Phys. Stat. Sol. 188, 293 (2001). doi:10.1002/1521-396X(200111)188:1<293::AID-PSSA29 3>3.0.CO;2-D

3. M.Y. Liao, Y. Koide, J. Alvarez, M. Imura and J. P. Kleider, Phys. Rev. B 78, 045112 (2008). doi:10.1103/PhysRevB. $\underline{78.045112}$

4. M. Y. Liao, J. Alvarez and Y. Koide, Appl. Phys. Lett. 90,123507 (2007). doi:10.1063/1.2715440

5. M. Y. Liao, Y. Koide and J. Alvarez, Appl. Phys. Lett. 87, 022105 (2005). doi:10.1063/1.1992660

6. Y. Koide, M. Y. Liao and J. Alvarez, Diamond Relat. Mater. 15, 1962 (2006). doi:10.1016/j.diamond.2006.08.009

7. M. Y. Liao and Y. Koide, Appl. Phys. Lett. 89, 113509 (2006). doi:10.1063/1.2349829

8. A. G. Gaydon, The Spectroscopy of Flames, Chapman and Hall, London, (1957).

9. J. Walker, Rep. Pro. Phys. 42, 1605 (1979). doi:10.1088/ $\underline{0034-4885 / 42 / 10 / 001}$ 